\title{
Causas do abandono ao tratamento da hipertensão de pacientes de uma unidade de saúde da família - Belém/PA
}

\author{
Causes of abandoning the treatment of hypertension in patients in a health unit family - \\ Belém/PA
}
Las causas de abandono en el tratamiento de la hipertensión en pacientes de una unidad de salud familiar - Belém/PA.

Marina Lopes de Freitas Freire ${ }^{1 *}$, Thaís Oliveira Santos ${ }^{1}$, Ismari Perini Furlaneto ${ }^{1,3}$, Osvaldo da Silva Peixoto ${ }^{1,2,3,4}$, Allen Washington Duarte Magalhães ${ }^{1}$, Marcello Oliveira Santos ${ }^{5}$, Natália Tavares Carvalho ${ }^{1}$, Nathália Jucá Azevedo Picanço ${ }^{1}$, Yanka Rafaela da Costa Neto Vieira ${ }^{1}$, Ana Karina Corôa Vasconcelos ${ }^{1}$, Israela Cristine Pereira Marinho ${ }^{1}$, Ana Luiza Sousa Barbosa ${ }^{1}$, Eduarda Teixeira Braga Bastos ${ }^{5}$, Maíra Khoury Evangelista ${ }^{5}$, Luiz Lima Chaves ${ }^{6}$.

\section{RESUMO}

Objetivo: Identificar causas de abandono ao tratamento de hipertensão e traçar o perfil sociodemográfico dos usuários de uma Unidade de Saúde da Família (USF). Metodologia: Trata-se de um estudo transversal descritivo e analítico, que investigou as possíveis causas de abandono ao tratamento para hipertensão oferecido pela USF Canal da Visconde entre de 01 de janeiro a 31 de dezembro de 2014, com aplicação de questionário estruturado a usuários cadastrados nessa USF e que tenham descontinuado o tratamento por um período igual ou superior a 60 dias. Resultados: Foram identificados 56 pacientes que atendiam os critérios de inclusão definidos, sendo a maioria do sexo feminino $(76,8 \%, p=0.0001)$, já haviam realizado o tratamento para hipertensão na USF $(78,6 \%, p<0.0001)$ e estavam sob responsabilidade dos profissionais da manhã $(85,7 \%, p<0.0001)$. O motivo principal do abandono ao tratamento por esses usuários foi possuírem plano de saúde (33,9\%) e fazerem acompanhamento em outro local $(28,6 \%) \quad(p=0.0093)$. Conclusão: $O$ sucesso do tratamento de hipertensão está diretamente relacionado com a adesão ao tratamento, sendo assim, os aspectos envolvidos no abandono devem ser amplamente investigados a fim de serem identificados e servirem de subsídios para minimizar a taxa de abandono.

Palavras-chave: hipertensão, adesão ao tratamento, serviços de saúde.

\footnotetext{
ABSTRACT

Objective: Identify the abandonment causes of the hypertension treatment and trace the socio-demographic profile of users in one Family Health Unit (FHU). Methodology: This is a descriptive and analytical crosssectional study, which investigated the possible abandonment causes treatment for hypertension offered by FHU Canal da Visconde between January 1 to December 31, 2014, with structured questionnaire to registered users that $\mathrm{FHU}$ and have discontinued the treatment for a period less than 60 days. Results: We identified 56 patients who met the inclusion criteria, and the majority were female $(76.8 \%, p=0.0001)$, had performed the treatment for hypertension at $\mathrm{FHU}(78.6 \%, \mathrm{p}<0.0001)$ and was the responsibility of the morning professionals

${ }^{1}$ Centro Universitário Metropolitano da Amazônia. Belém-PA. *E-mail: marinafreitasfreire@gmail.com

2Universidade Federal do Pará. Belém-PA.

3Universidade do Estado do Pará. Belém-PA.

${ }^{4}$ Pontifícia Universidade Católica do Rio de Janeiro. Rio de Janeiro-RJ.

${ }^{5}$ Centro Universitário do Estado do Pará. Belém-PA

6Universidade do Estado do Pará. Santarém-PA.
} 
$(85.7 \%, p<0.0001)$. The main reason for abandoning the treatment for these users is by having health insurance $(33.9 \%)$ and do follow-up elsewhere $(28.6 \%)(p=0.0093)$. Conclusion: The success of hypertension treatment is directly related to adherence to treatment, therefore, the issues involved in the abandonment should be fully investigated in order to be identified and serve as subsidies to minimize the dropout rate.

Key Words: hypertension, treatment adherence, health services.

\section{RESUMEN}

Objetivo: Identificar las causas del abandono del tratamiento de la hipertensión y trazar el perfil sociodemográfico de los usuarios de una Unidad de Salud de la Familia (USF). Metodología: Se trata de un estudio transversal, descriptivo y analítico, que investigó el posible abandono de el tratamiento de la hipertensión ofrecido por USF Canal visconde entre 1 de enero al 31 de diciembre de 2014 con la aplicacion de cuestionario estructurado para usuarios registrados en la USF y que han suspendido el tratamiento por un período inferior a 60 días. Resultados: Se identificaron 56 pacientes que cumplían los criterios de inclusión, la mayoría eran mujeres $(76,8 \%, p=0,0001)$, ya habían realizado el tratamiento para la hipertensión en la USF $(78,6 \%, p<0,0001)$ y estaban en la responsabilidad de los profesionales de la mañana $(85,7 \%, p$ $<0,0001)$. La razón principal para abandonar el tratamiento para estos usuarios es por tener un seguro de salud $(33,9 \%)$ y monitoreo la salud en otros lugares $(28,6 \%)(p=0,0093)$. Conclusión: El éxito del tratamiento de la hipertensión está directamente relacionada con la adherencia al tratamiento, por lo tanto, las cuestiones relacionadas con el abandono deben ser investigados a fondo con el fin de identificar y servir como subsidios para reducir al mínimo la tasa de abandono escolar.

Palabras clave: hipertensión, la adherencia al tratamento, causas del abandono.

\section{INTRODUÇÃO}

Nos últimos anos, ocorreu nas cidades brasileiras um aumento significativo no número de óbitos causados por doenças crônicas não transmissíveis, dentre elas a hipertensão arterial sistêmica (HAS). A HAS é uma doença crônica de origem multifatorial com elevada prevalência na população brasileira urbana, adulta e acomete mais da metade dos idosos no mundo, o que a torna fator determinante na mortalidade dessa população (BRASIL MS, 2005; VI DIRETRIZES BRASILEIRAS DE HIPERTENSÃO ARTERIAL, 2010)

Apesar de ser uma doença de baixa gravidade, caracteriza-se por sua cronicidade e pela pouca ou inexistência de sintomas específicos. Além de ser um fator de risco para eventos cardiovasculares, como: acidente vascular cerebral (AVC), doenças isquêmicas do coração (DIC), insuficiência renal e isquemia vascular periférica. $O$ controle de doenças crônicas é imprescindível, pois estas geram enormes danos socioeconômicos para o país, tornando-se um desafio para a saúde pública (LESSA I, 2006).

Através do Ministério da Saúde (MS), o país criou alguns Programas para o controle das doenças de maior impacto na população. Entre eles, foi criado o Hiperdia (Portaria no 371/GM, de 4 de março de 2002), por um Plano de Reorganização da Atenção à Hipertensão arterial sistêmica e Diabetes Mellitus, estabelecendo metas e diretrizes para ampliar ações de prevenção, diagnóstico, tratamento e controle dessas doenças, mediante a reorganização do trabalho de atenção à saúde. Trata-se de reuniões voltadas para hipertensos e diabéticos a fim de fornecer orientações sobre a doença, tendo como meta primordial o direcionamento das ações da equipe de saúde e a garantia do controle e adesão ao tratamento da HAS (GOMES TJO et al, 2010).

O controle da hipertensão faz-se por meio de tratamento medicamentoso contínuo além de mudanças no estilo de vida, como prática de atividade física e alimentação saudável, exigindo dos seus portadores controle durante toda a vida, o que dificulta a adesão ao tratamento. Os autores ainda citam que a adesão ao tratamento de qualquer doença crônica sofre influência de fatores comportamentais próprios do paciente; uma das grandes dificuldades do portador de hipertensão é entender porque devem utilizar diariamente diversos 
comprimidos e sofrer com efeitos colaterais para controlar um problema que não apresentou sintomas (SOUSA MF e HAMANN EM, 2009; GOMES TJO et al, 2010; CONTIERO AP et al, 2009). Deste modo, como forma de melhorar a adesão terapêutica, torna-se extremamente importante o estabelecimento de relações confiáveis entre o usuário e os profissionais da saúde já que a mesma é ponto fundamental para o planejamento de uma gestão de saúde qualificada (MARTINS CM e STAUFFER AB, 2007; GUSMÃO JL et al, 2009).

A aferição regular da pressão arterial, comparecimento ou não às consultas médicas e de enfermagem e interrupção do tratamento medicamentoso são variáveis objetivas que podem indicar o grau de abandono dos hipertensos ao tratamento, no qual apenas $40 \%$, aproximadamente, conseguem manter os níveis de pressão arterial controlados (BORGES PCS e CAETANO JC, 2005; KING DS et al, 2003).

Não existem muitos estudos, no Brasil e no mundo, que descrevem índices de adesão entre os pacientes hipertensos, isso indica uma maior necessidade de investigações no intuito de orientar a equipe de saúde da família e usuários ao comportamento adequado em relação a esse problema (GOMES TJO et al, 2010, BARBOSA RGB e LIMA NKC, 2006; DOSSE C et al, 2009).

Faz-se necessário, portanto, a identificação e o desenvolvimento de possíveis mecanismos que retenham ou atraiam pacientes para os programas de controle de hipertensão, como forma de minimizar os possíveis danos ao paciente e o aumento de gastos públicos com essa doença.

O objetivo deste estudo foi identificar as causas de abandono ao tratamento de hipertensão disponibilizados na USF, quantificando-as, e traçar o perfil sociodemográfico do pesquisado.

\section{MÉTODOS}

A pesquisa constitui-se em um estudo transversal descritivo, no qual foram incluídos 80 pacientes hipertensos cadastrados na Unidade de Saúde da Família Canal da Visconde, em Belém, Pará, Brasil e que abandonaram o tratamento para HAS.

Após aprovação do Comitê de Ética em pesquisa do Centro Universitário do Maranhão (UNICEUMA), de número 1.368.515, iniciou-se a coleta de dados que foi dividida em dois momentos.

No primeiro momento, realizou-se levantamento de dados no formulário de atendimento da USF, no qual 131 usuários hipertensos cadastrados não eram assíduos ao programa Hiperdia no período de 01 de janeiro a 31 de dezembro de 2014.

No total foram selecionados 80 pacientes, visto que os outros 51 não se encontravam mais na área de abrangência da USF.

Entretanto, devido os critérios de inclusão serem o abandono ao tratamento de hipertensão oferecido pela USF Canal da Visconde ou o não comparecimento às consultas durante dois meses consecutivos, apenas 56 pacientes se enquadraram no estudo.

No segundo momento, foi realizada busca ativa com aplicação de questionário entre os usuários faltosos, para a identificação dos motivos do seu abandono ao tratamento e traçar o perfil sociodemográfico desses pacientes.

$\mathrm{Na}$ análise dos dados referentes às características sociodemográficas foram tratados utilizando estatística descritiva e expressos sob a forma de média \pm desvio padrão, Intervalo de Confiança (95\%) e frequências absoluta e relativa, conforme o caso, e apresentados em tabelas e/ou gráficos.

As frequências observadas em cada categoria foram comparadas com as esperadas ao acaso utilizando o teste do Qui-Quadrado de Aderência (ou teste G de Aderência, conforme a necessidade).

Todos os testes foram executados com o auxílio do software BioEstat 5.4 e resultados com $p \leq 0.05$ (bilateral) foram considerados significativos. 


\section{RESULTADOS e DISCUSSÃO}

Foram identificados, durante o período do estudo, 56 pacientes que atendiam aos critérios de inclusão definidos, os quais foram convidados a participar da presente pesquisa. Destes, a maioria (43/56,76.8\%; $p=$ $0.0001)$ era do sexo feminino, já realizou o tratamento para HAS na USF Canal da Visconde (44/56, 78.6\%; $p<0.0001$ ), e estavam - à época do acompanhamento - sob responsabilidade dos profissionais da equipe da manhã (48/56, 85.7\%; $p<0.0001)$. A idade média dos participantes era de 65.4 anos $( \pm 12.5$ anos), variando entre 32 e 86 anos (IC95\% 62.2 - 68.7) e todos se consideravam hipertensos (Figura 1).

Tabela 1 - Perfil socioepidemiológico dos usuários cadastrados na Unidade se Saúde da Família Canal da Visconde que abandonaram o tratamento para hipertensão.

\begin{tabular}{lcc}
\hline Característica & $\boldsymbol{n}(\%)$ & $\boldsymbol{p}^{\text {-valor }}{ }^{\star}$ \\
\hline Gênero & $43(76.8)$ & \\
$\quad$ Feminino & $13(23.2)$ & $0.0001^{\dagger}$ \\
Masculino & & \\
Escolaridade & $30(53.6)$ & $<0.0001^{\dagger}$ \\
$\quad$ Ensino Fundamental (completo/incompleto) & $22(39.3)$ & \\
Ensino Médio (completo/incompleto) & $04(7.10)$ & \\
Ensino Superior (completo/incompleto) & $09(16.0)$ & \\
Estado civil & $30(53.6)$ & \\
$\quad$ Solteiro & $17(30.4)$ & \\
Casado & & \\
Separado ou viúvo & $01(1.80)$ & \\
Já realizou tratamento na USF & $44(78.6)$ & \\
Sim, faz atualmente & $11(19.6)$ & \\
Sim, mas abandonou & & \\
Não, nunca fez &
\end{tabular}

Legenda: *Qui-Quadrado de Aderência. ${ }^{\dagger}$ Estatisticamente significativo.

Fonte: Protocolo de pesquisa 2016.

Quanto às razões pelas quais estes usuários atualmente abandonaram o tratamento da HAS na USF Canal da Visconde, a minoria alegou como principal motivo a impossibilidade de se deslocar até a USF (04/56, $7.1 \%$ ) e os motivos mais frequentemente relatados como principais foram "possuir plano de saúde" e "fazer o acompanhamento em outro local", referidos por $33.9 \%(19 / 56)$ e $28.6 \%$ (16/56) dos entrevistados, respectivamente $(p=0.0093)$ (Gráfico 1$)$.

Gráfico 1 - Distribuição dos usuários cadastrados no Programa Hiperdia da Unidade de Saúde da Família Canal da Visconde, segundo o principal motivo pelo qual atualmente não fazem o tratamento nesta USF.

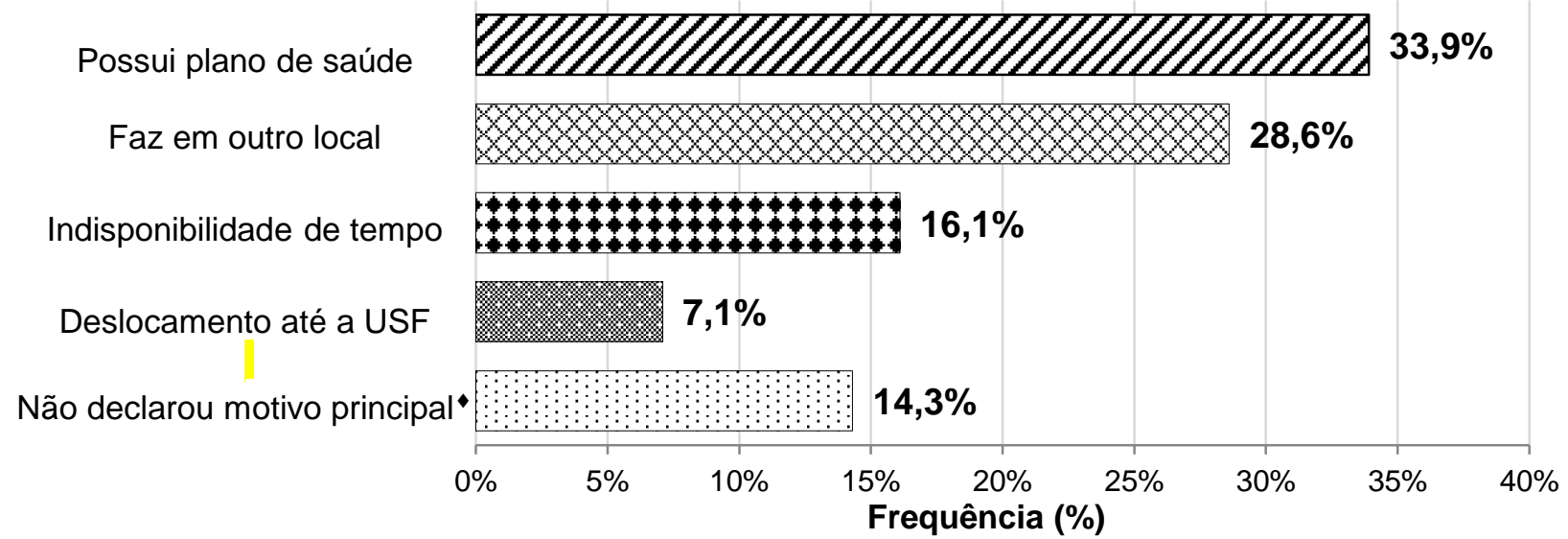

Fonte: Protocolo de pesquisa 2016. $p=0.0093^{\dagger}$ (Qui-Quadrado de Aderência). ${ }^{\dagger}$ Estatisticamente significativo. •Desconsiderado na análise estatística. 
Dos 56 usuários entrevistados, apenas 20 (35.7\%) esclareceram as razões que motivaram o abandono do tratamento na referida USF e/ou os fizeram buscar alternativas para tratar a HAS. Dentre os fatores citados, sete pacientes $(35 \%)$ consideravam desnecessário o acompanhamento - apesar de saberem-se hipertensos -, seis (30\%) alegaram fatores relacionados à equipe da USF (atendimento ruim e/ou falta de visita domiciliar do médico ou outros membros da equipe), quatro (20\%) citaram razões associadas à própria USF, como falta de medicamentos, necessidade de outros especialistas ou falta de médico no turno em que está alocado, e três (15\%) alegaram razões pessoais, como indisponibilidade de tempo por trabalhar e/ou cuidar de familiar $(p=0.5794)($ Gráfico 2).

Gráfico 2 - Distribuição dos usuários cadastrados no Programa Hiperdia da Unidade de Saúde da Família Canal da Visconde, segundo as razões que motivaram o abandono do tratamento nesta USF.
Não declarou motivo principal
Deslocamento até a USF
Indisponibilidade de tempo
Faz em outro local
Possui plano de saúde

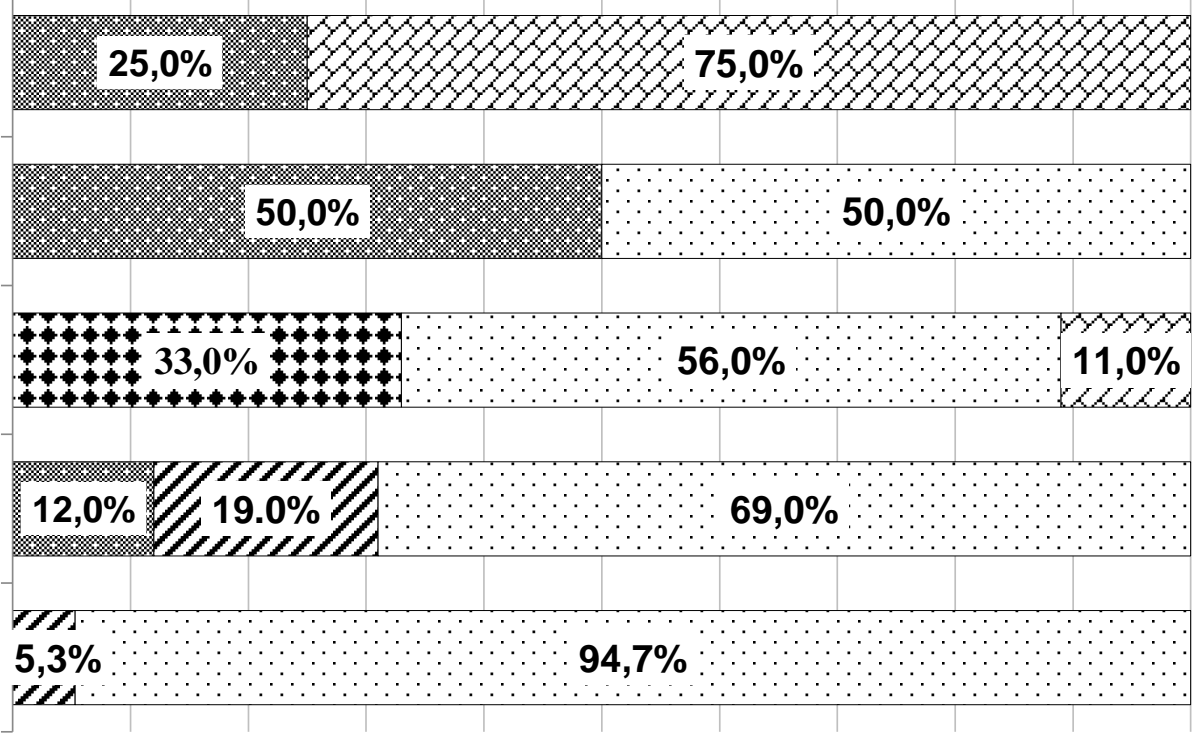

Frequência (\%)

\author{
Razões relacionadas à Equipe \\ $\square$ Razões relacionadas à USF \\ - Motivos pessoais \\ $\square$ Não informou \\ ๑Declara-se assintomático/Não acha necessário
}

$p=0.5794$ (Teste G de Aderência).

Fonte: Protocolo de pesquisa 2016

No presente estudo, notou-se que a maioria dos pacientes que abandonaram o tratamento era do sexo feminino, com idade média de 65,4 anos, baixa escolaridade e casados. Tradicionalmente, a HAS é mais frequente em homens com até 50 anos, e a presença de mais mulheres com diagnóstico de HAS pode sugerir o fato de as mulheres procurarem mais os serviços de saúde, determinando o diagnóstico da doença (VANCINI-CAMPANHARO CR et al, 2015)

É válido ressaltar as limitações encontradas durante a pesquisa, como a falta de informações contidas nas fichas de atendimento - que se apresentavam quase sempre incompletas, além da grande dificuldade em localizar a residência dos usuários selecionados, fazendo com que os pesquisadores necessitassem do auxílio dos agentes comunitários de saúde (ACS) para chegar às casas, o que determinou maior disponibilidade de tempo para a coleta de dados. Além disso, algumas microáreas da população adscrita se encontravam em setores com altos índices de assaltos e outros tipos de violência, o que exigiu ainda mais a 
presença de uma pessoa que possuía conhecimento do território, no caso, o ACS, além da necessidade adicional de realizar essas visitas em horários mais oportunos.

Suspeitava-se, no início do estudo, que as causas de abandono estivessem relacionadas ao fato de os usuários realizarem o tratamento em outro local. De fato, após a coleta de dados e posterior análise, verificouse que a maioria dos indivíduos que abandonou o tratamento, na amostra avaliada, referiu tê-lo descontinuado na USF Canal da Visconde por possuírem plano de saúde, corroborando com a hipótese inicialmente elaborada.

No que diz respeito ao abandono do paciente ao tratamento anti-hipertensivo, notou-se que, apesar de conhecerem sua situação quanto à doença, 35\% (07/20) dos pacientes consideravam desnecessário manter o acompanhamento, o que sugere que estas pessoas não entendem a doença como um problema de saúde que necessita de tratamento, talvez pelo fato de serem assintomáticos. Esse comportamento pode estar associado à baixa condição financeira e ao baixo nível de escolaridade dos pesquisados.

Segundo Osamor e Owumi (2011) o baixo nível de conhecimento sobre a doença, a falta de orientação adequada, os aspectos socioeconômicos, assim como o estresse e a ansiedade são associados a não adesão e ao abandono do tratamento.

Além disso, alguns autores citam que a elevação da escolaridade aumenta a adesão ao tratamento da hipertensão e que o nível educacional tem sido apontado como o fator socioeconômico mais importante no estado de saúde, principalmente na saúde cardiovascular (SILVA AP et al, 2016; NUNES MGS et al, 2015)

Dentre as características que definiram o perfil da amostra avaliada, observou-se que a idade média dos participantes, em 95\% das vezes, situou-se acima dos 60 anos, sendo esta faixa etária a de maior risco, concordando com as estimativas descritas pela VI Diretrizes Brasileiras de Hipertensão Arterial, a qual estimou que mais de $60 \%$ dos idosos são hipertensos (DOSSE C et al, 2009).

Quanto ao estado civil, o fato de morar com o companheiro foi identificado em alguns estudos como um fator de adesão ao tratamento, os quais observaram que hipertensos que moravam acompanhados apresentaram incremento de $145 \%$ na adesão ao tratamento. Entretanto, no presente estudo, mais da metade das pessoas que abandonaram o tratamento eram casadas, contradizendo o que era esperado pelos pesquisadores (NUNES MGS et al, 2015).

Outro fator de grande relevância percebido pelos pesquisadores foi acerca da discrepância das condições socioeconômicas entre as microáreas. Este fato foi notado por conta de $36.9 \%$ da amostra possuir plano de saúde e $28.6 \%$ realizar o acompanhamento em outro local.

Durante as entrevistas, também chamou a atenção dos pesquisadores a constatação de que grande parte dos usuários alegou procurar realizar tratamento em outros locais por possuírem maior disponibilidade de especialistas em relação à USF.

Segundo Martins et al (2014) a falta de medicamentos nas unidades também é um fator determinante associado à não adesão ao tratamento, o que foi uma situação relatada pela população em estudo, dentro da qual se enquadra em razões associadas à própria USF, que foi a alternativa escolhida por estes, no momento em que o questionário foi aplicado.

Além disso, 30\% dos pesquisados alegaram fatores relacionados à equipe da USF, como atendimento ruim e/ou falta de visita domiciliar do médico, visto que não tinham como se deslocar até à USF (OSAMOR PE e OWUMI BE, 2011)

Um dado preocupante encontrado no estudo foi o fato de que, por não serem sintomáticos, os usuários não consideravam necessária a continuidade do tratamento. Isso chama a atenção para a necessidade de se formular estratégias mais eficientes de educação em saúde por parte da equipe multiprofissional, de forma que garantam a clareza na transmissão de informações para o usuário e a consequente continuidade do tratamento, pois o alcance deste objetivo não depende unicamente do paciente, mas de todos os elementos que permeiam este processo (OSAMOR PE e OWUMI BE, 2011). 


\section{CONCLUSÃO}

O sucesso do tratamento de HAS está diretamente relacionado com a sua adesão. Os resultados obtidos no estudo permitem sugerir que causas básicas como falta de medicamentos e a falta de um bom relacionamento entre os usuários e a equipe multiprofissional, podem repercurtir na qualidade de vida dessas pessoas. A caracterização da não adesão e do abandono aos tratamentos da HAS constitui um grande desafio tanto para o Estado como também para os trabalhadores de saúde. Esses achados podem proporcionar subsídios para a realização de intervenções eficientes na Atenção Primária a esses pacientes.

\section{REFERÊNCIAS}

1. BARBOSA RGB, LIMA NKC. Índices de adesão ao tratamento anti-hipertensivo no Brasil e mundo. Rev Bras Hipertens 2006; Vol. 13(1): 35-38.

2. BORGES PCS, CAETANO JC. Abandono do tratamento da hipertensão arterial sistêmica dos pacientes cadastrados no hiperdia/MS em uma unidade de saúde do município de Florianopolis-SC. Arquivos Catarinenses de Medicina 2005; Vol. 34:45-50.

3. BRASIL MS. A vigilância, o controle e a prevenção das doenças crônicas não transmissíveis: DCNT no contexto do Sistema Único de Saúde brasileiro. Brasília: Organização Pan-Americana de Saúde; 2005.

4. CONTIERO AP, et al. Idoso com hipertensão arterial: dificuldades de acompanhamento na Estratégia Saúde da Família. Rev Gaúcha Enferm., Porto Alegre (RS) 2009 mar; 30(1): 62-70.

5. DIRETRIZES BRASILEIRAS DE HIPERTENSÃO ARTERIAL. Sociedade Brasileira de Cardiologia/Sociedade Brasileira de Hipertensão/Sociedade Brasileira de Nefrologia. Arq Bras Cardiol. 2010; 95(1): 1-51.

6. DOSSE C, et al. Fatores associados à não adesão dos pacientes ao tratamento de hipertensão arterial. Rev Latinoam Enfermagem 2009 mar-abr; 17(2). www.eerp.usp.br/rlae.

7. GOMES TJO, et al. Controle da pressão arterial em pacientes atendidos pelo programa hiperdia em uma unidade de saúde da família. Rev Bras Hipertens 2010; 17(3): 132-9.

8. GUSMÃO JL, et al. adesão ao tratamento em hipertensão arterial sistólica isolada. Rev Brás Hipertens 2009; 16(1): 38-43.

9. KING DS, et al. Medication adherence in a southeastern hypertension clinic. Am J Hypertens. 2003; 16(5): 234A.

10. LESSA I. Impacto social da não-adesão ao tratamento da hipertensão arteria. Rev Bras Hipetens 2006; Vol. 13(1): 39-46.

11. MARTINS AG, et al. Adesão ao tratamento clínico ambulatorial da hipertensão arterial sistêmica. Acta paul. enferm; 2014; 27(3): 266-27.

12. MARTINS CM, STAUFFER AB. Educação e Saúde. Rio de Janeiro: EPSJV/ Fiocruz; 2007.

13. NUNES MGS, et al. Prevalência e Fatores Associados a Cooperação do Paciente Portador de Hipertensão Arterial. Acta Paul Enferm 2015; 28(4):323-30.

14. OSAMOR PE, OWUMI BE. Factors associated with treatment compliance in hypertension in southwest Nigeria. $J$ Health Popul Nutr. 2011; 29(6): 619-28.

15. SILVA AP, et al. Fatores associados à não adesão ao tratamento da hipertensão arterial sistêmica: uma revisão integrativa. J Res.: fundam care online 2016 jan-mar; 8(1):4047-4055.

16. SOUSA MF, HAMANN EM. Programa Saúde da Família uma agenda incompleta? Cien saúde Colet $2009 ; 14$ (Supl. 1): 1325-1335.

17. VANCINI-CAMPANHARO CR, et al. Hipertensão Arterial Sistêmica no Serviço de Emergência: adesão medicamentosa e conhecimento da doença. Rev Latino-Am Enfermagem 2015 nov-dez; 23(6): 1149-56. 